\title{
Some Algebraic Structures Arising From Black-box Automata
}

\author{
Jānis CĪRULIS \\ Institute of Mathematics and Computer Science, University of Latvia, \\ Raina b., 29, Riga LV-1050, Latvia \\ janis.cirulis@lu.lv
}

\begin{abstract}
A representative example of a black-box automaton is provided by a (possibly nondeterministic) automaton with any information about its states ignored. Such an automaton realizes a certain many-valued transformation $\varphi$ of input strings available to it into output strings. The outcome space of a black-box automaton is defined to be the set of all pairs $(\alpha, \gamma)$ where $\alpha$ is its input string, and $\gamma \in \varphi(\alpha)$ is any of the corresponding output strings. We show that each outcome space carries a structure of a tree-ordered poset equipped with two special binary relations and that there is, up to isomorphisms, a bijective correspondence between such relational structures and the so called exact black-box automata. Moreover, an otcome space is also a bisemigroup of certain kind. Virtual states of a black-box automaton also are discussed.
\end{abstract}

Keywords: automaton; bisemigroup; black box; ND-operator; overriding; outcome space; tree; virtual state

\section{Introduction}

Let $\Sigma$ be some nonempty set considered as an alphabet. As usual, where $M$ is a subset of $\Sigma, M^{*}$ stands for the set of all finite strings (words) over $M$, including the empty string $o$. The notation $\alpha \leq \beta$ for $\alpha, \beta \in \Sigma^{*}$ means that $\alpha$ is a prefix of $\beta$. The symbol $|\alpha|$ stands for the length of a string $\alpha$. The set of all strings of length $|\alpha|$ is denoted by $\Sigma^{\alpha}$. If $|\alpha| \leq|\beta|$, then $\beta \mid \alpha$ is the prefix of $\beta$ of length $|\alpha|$, and, for $L \subseteq \Sigma^{\beta}$, $L \mid \alpha:=\{\gamma \mid \alpha: \gamma \in L\}$. In particular, $\Sigma^{\alpha}=\Sigma^{\beta} \mid \alpha$, and $L \mid \alpha=\varnothing$ when $L=\varnothing$.

Suppose that $X, Y \subseteq \Sigma$ and that there is some black box $\mathbf{B}$ with one input and one output which transforms strings from $X^{*}$ into strings from $Y^{*}$ working in a discrete time in the following way ("on-line"): when symbols of an input string $\alpha$ are sequentially presented to the black box, it processes each symbol and produces, also sequentially and "without anticipation", symbols forming an output string $\gamma$. In particular, $\gamma$ is always of the same length as $\alpha$. Such a procedure may be considered as a simple experiment with the black box (Moore, 1956), and the pair $(\alpha, \gamma)$ can then be taken as 
an outcome of the experiment. We also admit that the response to any given input string is not necessary uniquely determined by the latter (for reasons unspecified here), but, for the present, exclude deadlocks. Summing up, this means that $\mathbf{B}$ realizes a so called sequential ND-operator over $[X, Y]$ (see (Starke, 1969), Definition II.4.3); 'ND' stands for 'non-deterministic'), i.e., a totally defined multi-valued mapping $\varphi$ of $X^{*}$ into $Y^{*}$ such that

(i) if $\alpha \in X^{*}$, then $\varphi(\alpha) \subseteq Y^{\alpha}$, and

(ii) if $\alpha \leq \gamma \in Y^{*}$, then $\varphi(\alpha)=\varphi(\gamma) \mid \alpha$.

We denote by $\mathcal{P}_{0}(M)$ the set of nonempty subsets of a set $M$ and identify singleton subsets with their single elements; thus $\varphi$ may be treated as a function $X^{*} \rightarrow \mathcal{P}_{0}\left(Y^{*}\right)$.

One may try to explain multivalence of $\varphi$ assuming that the black box has some set of states and that it may be in any of these states at the beginning of an experiment. Moreover, the states themselves could be non-deterministic, as in the example below. Generally, any information that allows to diminish the uncertainty in the behavior of $\mathbf{B}$ may be interpreted as a state of $\mathbf{B}$. We shall not need, however, any assumption concerning states up to Section 3 .

Example. Instances of a black box of this kind are provided by any nondeterministic Mealy automaton-a quintuple $\mathbf{A}:=(X, Y, Z, \delta, \lambda)$, where $X, Y \subseteq \Sigma$ and

- $X$ is a set of input symbols,

- $Y$ is a set of output symbols,

- $Z$ is a set of states,

- $\delta$ is a many-valued next-state function $X \times Z \rightarrow \mathcal{P}_{0}(Z)$,

- $\lambda$ is a many-valued output function $X \times Z \rightarrow \mathcal{P}_{0}(Y)$,

(cf. Definition II.1.5 in (Starke, 1969)). The behaviour function of $\mathbf{A}$ in a state $z$ is a function $\Lambda_{z}: X^{*} \rightarrow \mathcal{P}_{0}\left(Y^{*}\right)$ defined as follows: $\Lambda_{z}(\alpha)$ is the set of all output strings A can produce, being in the state $z$, in response to the input string $\alpha$. More precisely, $\Lambda_{z}(o)=o$ and, for all $x_{1}, x_{2}, \ldots, x_{m} \in X$ and $y_{1}, y_{2}, \ldots, y_{n} \in Y$,

$$
\begin{aligned}
& y_{1} y_{2} \cdots y_{n} \in \lambda_{z}\left(x_{1} x_{2} \cdots x_{m}\right) \text { if and only if } \\
& \qquad n=m \text { and, for all } i=1,2, \ldots m, y_{i} \in \lambda\left(x_{i}, z_{i}\right),
\end{aligned}
$$

where $z_{1}=z$ and $z_{i+1}$ is any state from $\delta\left(x_{i}, z_{i}\right)$.

Setting, for a nonempty set of states $M, \Lambda_{M}(\alpha):=\bigcup\left(\Lambda_{z}(\alpha): z \in M\right)$, we obtain the behaviour function $\Lambda_{M}$ of $\mathbf{A}$ in a "macrostate" $M$ (and of $\mathbf{A}$ as a whole if $M=Z$ ). It is always a sequential ND-operator over $[X, Y]$, and so any triple $\left(X^{*}, \Lambda_{M}, Y^{*}\right)$ is in fact a paticular black box. See Part II of (Starke, 1969) for more information on ND-automata and sequential ND-operators.

Every black box $\mathbf{B}$ can be realized in this way by an ND-automaton, as the following proposition shows. This suggests a term 'black-box automaton' introduced in Section 2 for slightly more abstract black boxes.

Proposition 1.1 ((Starke 1969), Satz II.4.6) To every sequential ND-operator $\varphi$ over $[X, Y]$, there are an ND-automaton $\mathbf{A}$ over $\left[X^{\prime}, Y^{\prime}\right]$ with $X \subseteq X^{\prime}$ and a nonempty subset $M$ of its states such that $\varphi(\alpha)=\Lambda_{M}(\alpha)$ for all $\alpha \in X^{*}$. 
As noted above, a pair $(\alpha, \gamma)$ with $\alpha \in X^{*}$ and $\gamma \in \varphi(\alpha)$ may be thought of as an outcome of an experiment with the black box. We call the set $\Phi$ of all such pairs (which actually is the graph of $\varphi$ ) the outcome space of $\mathbf{B}$. Our purpose in the paper is investigation of the structure of the outcome spaces of black boxes (black-box automata).

In the next section, we give a formal definition of black-box automaton and ascertain that the outcome space $\Phi$ of such an automaton is a tree-ordered poset equipped with an additional relation, called overriding in (Cîrulis, 2002). In Section 3, the so called virtual states of a black-box automaton are defined essentially as certain ND-operators similar to the behavior functions $\Lambda_{M}$ in the above example; it is shown here that the virtual states form a join semilattice isomorphic to a semilattice of some special subsets of $\Phi$. The main result of Section 4 states that every tree-ordered poset with overriding is isomorphic to the outcome space of some black-box automaton; this is a consequence of a general construction theorem for a black-box automaton from such a poset. However, the representing automation is not unique (even up to isomorphism), which means that the description of outcome spaces obtained in Section 2 is still too weak. In Section 5, discussed are tree-ordered posets with two overriding relations interrelated in a certain way. Outcome spaces are shown to have even such expanded structure, and it is proved that two black-box automata are isomorphic if and only if their outcome spaces are isomorphic as structures of this kind. Finally, in Section 6, a class of certain tree-ordered bisemigroups - sets equipped with two semigroup operations is considered. Any blackbox automaton yields a bisemigroup (its outcome space) which belongs to this class, and every bisemigroup from this class gives rise to a pair of overriding relations interrelated as in Section 5.

Concluding the section, we mention one more derived structure of a black box that deserves an interest. Given a black box $\mathbf{B}$, consider a pair $(\alpha, K)$, where $\alpha$ is its input string and $K$ is a subset of $\varphi(\alpha)$, the set of possible responses to $\alpha$. It may be associated with an experimental statement about outcomes of $\mathbf{B}$ associated with an input string $\alpha$ and is true of an outcome $(\alpha, \gamma)$ if and only $\gamma \in K$. The set of all such pairs may be called a statement space of $\mathbf{B}$. Its structure will be investigated in another paper, which is in preparation.

\section{Black-box automata and their outcome spaces}

The model of a black box described in Introduction includes some specific details which are unrelevant for our purposes and make it "too concrete". We first slightly modify the model to give it a more algebraic form.

The sets of input and of output strings of a black box, $X^{*}$ and $Y^{*}$ respectively, support the structure of monoid (w.r.t. the concatenation), but they naturally are also rooted trees. For our purposes, the tree structure of these sets is more important. Algebraically, a rooted tree may be treated as a poset $(T, \leq)$ with bottom in which every initial segment is a finite chain. It is then easily seen that $T$ is even a lower semilattice, i.e., any pair of its elements has the greatest lower bound, or meet. Generally, elements of the poset are interpreted as nodes of a tree, and $s \leq t$ for $s, t \in T$ then means that $s$ lies on the path from the bottom $\perp$ to $t$. (There is also another, the so called CPT interpretation, when elements of the poset are considered as paths of a tree, and the order $\leq$, as inclu- 
sion of paths.) The set $\Sigma^{*}$ and its subsets $X^{*}$ and $Y^{*}$ become algebraic trees in a more direct way — when equipped with the relation '... is a prefix of ...' (see Introduction); then the greatest common prefix of two strings is their meet. Any nonempty subset of $T$ which contains together with an element of $T$ also all elements it dominates is called a subtree of $T$; so $X^{*}$ and $Y^{*}$ are subtrees of $\Sigma^{*}$.

By now on, by a tree we mean an algebraic rooted tree as explained above-a "treeordered poset", i.e., a poset with a least element where all initial segments are finite chains. Every such a tree $T$ can be embedded into a tree of strings, say, $\Sigma^{*}$, i.e., is isomorphic to a subtree of $\Sigma^{*}$, for a sufficiently large $\Sigma$ (the size of $\Sigma$ is determined by the supremum of degrees (numbers of the successors) of all elements in $T$ ). However, it will be more convenient to work with the abstract trees. We adapt to them the notation introduced for $\Sigma^{*}$ at the beginning of the previous section. In particular, $|t|$ now denotes the height of an element $t \in T$, i.e., the number of elements in the chain $[\perp, t]$ minus 1 , where $\perp$ stands for the bottom of $T$. (In $\Sigma^{*}$, the height of a string is equal to its length.) The set $\{t \in T:|t|=|s|\}$ is denoted by $T_{s}$. If $|s| \leq|t|$, then the symbol $t \mid s$ stands for the single element of height $|s|$ in the chain $[\perp, t]$, and if $U \subseteq T_{t}$, then $U \mid s:=\{u \mid s: u \in U\}$.

We now assign a refined meaning to a term usually used informally (and in differing senses).

Definition 2.1. A black-box automaton is a triple $\mathbf{B}:=(I, \varphi, O)$, where

- $I$ is a tree (the input tree),

- $O$ is a tree (the output tree),

- $\varphi$ is a function (the behavior of $\mathbf{B}) I \rightarrow \mathcal{P}_{0}(O)$ such that

(i) if $\alpha \in I$, then $\varphi(\alpha) \subseteq O_{\alpha}$, and

(ii) if $\alpha \leq \beta \in I$, then $\varphi(\alpha)=\varphi(\beta) \mid \alpha$.

The graph $\Phi:=\{(\alpha, \gamma): \alpha \in I, \beta \in \varphi(\alpha)\}$ of $\varphi$ is called the outcome space of $\mathbf{B}$. The automaton B is called exact if the range $\varphi(I)$ of $\varphi$ coincides with $O$.

Evidently, the model of a black box discussed in Introduction fits in with this definition. In $I$ and $O$, we write $o$ for the least element. Clearly, $\varphi(o)=o$, and if $(\alpha, \gamma) \in \Phi$, then $|\alpha|=|\gamma|$. The range of $\varphi$ is generally a subtree of $O$; so, exactness is not a sever restriction. Notice that trees having elements (nodes) with different degrees (numbers of successors) are not excluded; in particular, a node in $I$ may have not any successor at all. Thus, deadlocks also can be simulated in a black-box automaton.

The set $\Phi$ is ordered by the componentwise defined relation $\leq$ :

$$
(\alpha, \gamma) \leq(\beta, \delta): \equiv \alpha \leq \beta \text { and } \gamma \leq \delta
$$

and $(o, o)$ is its bottom element. The inequality $(\alpha, \gamma) \leq(\beta, \delta)$ is naturally interpreted as saying that the outcome $(\alpha, \gamma)$ is a part of, or included in, the outcome $(\beta, \delta)$.

Lemma 2.2 The poset $(\Phi, \leq,(o, o))$ is a tree with meets characterized by

$$
\left(\alpha_{1}, \gamma_{1}\right) \wedge\left(\alpha_{2}, \gamma_{2}\right)=\left\{\begin{array}{l}
\left(\alpha_{1} \wedge \alpha_{2}, \gamma_{2} \mid\left(\alpha_{1} \wedge \alpha_{2}\right) \text { if }\left|\alpha_{1} \wedge \alpha_{2}\right| \leq\left|\gamma_{1} \wedge \gamma_{2}\right|\right. \\
\left(\alpha_{1} \mid\left(\gamma_{1} \wedge \gamma_{2}\right), \gamma_{1} \wedge \gamma_{2}\right) \text { if }\left|\alpha_{1} \wedge \alpha_{2}\right| \geq\left|\gamma_{1} \wedge \gamma_{2}\right|
\end{array}\right.
$$


Proof. As noticed, $(o, o)$ is a bottom element in $\Phi$. Let $(\beta, \delta)$ be a pair in $\Phi$. If $\left(\alpha_{1}, \gamma_{1}\right)$, $\left(\alpha_{2}, \gamma_{2}\right) \leq(\alpha, \gamma)$, then $\alpha_{1}, \alpha_{2} \leq \beta$, so that $\alpha_{1}$ and $\alpha_{2}$ are comparable (as $I$ is a tree); say, $\alpha_{1} \leq \alpha_{2}$. Likewise, $\gamma_{1}$ and $\gamma_{2}$ are comparable; then, of course, $\gamma_{1} \leq \gamma_{2}$ (as $\left|\alpha_{1}\right|=\left|\gamma_{1}\right|$ and $\left.\left|\alpha_{2}\right|=\left|\gamma_{2}\right|\right)$ and, further, $\left(\alpha_{1}, \gamma_{1}\right) \leq\left(\alpha_{2}, \gamma_{2}\right)$. Thus the initial segment of $\Phi$ determined by $(\beta, \delta)$ is a chain. The chain is finite, as the height of $(\beta, \delta)$ equals to $|\beta|$ (or $|\delta|$, what amounts to the same). So $\Phi$ is tree-ordered.

Finally, let $\left(\alpha_{1}, \gamma_{2}\right),\left(\alpha_{2}, \gamma_{2}\right)$ by any elements of $\Phi$. Suppose that $\left|\alpha_{1} \wedge \alpha_{2}\right| \leq$ $\left|\gamma_{1} \wedge \gamma_{2}\right|$. Then $\gamma_{1}\left|\left(\alpha_{1} \wedge \alpha_{2}\right)=\gamma_{2}\right|\left(\alpha_{1} \wedge \alpha_{2}\right)$. Consequently, $\left(\alpha_{1} \wedge \alpha_{2}, \gamma_{2} \mid\left(\alpha_{1} \wedge \alpha_{2}\right)\right)$ is a lower bound of $\left(\alpha_{1}, \gamma_{1}\right)$ and $\left(\alpha_{2}, \gamma_{2}\right)$. If $(\beta, \delta)$ is one more lower bound, then $\beta \leq \alpha_{1}, \alpha_{2}$ and $\beta \leq \alpha_{1} \wedge \alpha_{2}$. Likewise, $\delta \leq \gamma_{1}, \gamma_{2}$; moreover, $|\delta|=|\beta| \leq\left|\alpha_{1} \wedge \alpha_{2}\right|$. Then $\delta \leq \gamma_{1}\left|\left(\alpha_{1} \wedge \alpha_{2}\right), \gamma_{2}\right|\left(\alpha_{1} \wedge \alpha_{2}\right)$ and, further, $(\beta, \delta) \leq\left(\alpha_{1} \wedge \alpha_{2}, \gamma_{2} \mid\left(\alpha_{1} \wedge \alpha_{2}\right)\right)$, as required. The opposite case $\left|\alpha_{1} \wedge \alpha_{2}\right| \geq\left|\gamma_{1} \wedge \gamma_{2}\right|$ is treated similarly.

Two outcomes $(\alpha, \gamma)$ and $(\beta, \delta)$ may be considered as alternative if $\alpha=\beta$, and the first of them, as overridden in a sense by the second one if $\alpha \leq \beta$. Let us take a look on these relationships in a more abstract setting.

Digression 1. Let $(P, \leq)$ be a poset. Adapting Definition 2.2 from (Cīrulis, 2002), we say that a preorder relation $\sqsubseteq$ on $P$ is an overriding relation (an inclusion ' $p \sqsubseteq q$ ' being read as ' $p$ is overridden by $q$ ') if it satisfies the axioms

$$
\begin{gathered}
\text { (i) if } p \leq q \text {, then } p \sqsubseteq q \text {, (ii) if } p \sqsubseteq q \text { and } p, q \leq r \text {, then } p \leq q \text {, } \\
\text { (iii) if } p \sqsubseteq r \text {, then } p \| q \leq r \text { for some } q,
\end{gathered}
$$

where $\|$ is the equivalence relation induced by the preorder $\sqsubseteq$ (i.e., $p \| q$ iff $p \sqsubseteq q$ and $q \sqsubseteq p)$, and

$$
\text { (iv) if } p, q \sqsubseteq r \text { and } p \vee q \text { exists, then } p \vee q \sqsubseteq r \text {; }
$$

this axiom is superfluous in trees and may be omitted in the present context. It is immediate from (ii) that the element $q$ in (iii) is unique; let us denote it by $p b r$ and consider as a projection of $r$ onto $p$.

Let $(P, \leq, \sqsubseteq)$ be a poset with overriding, or just an o-poset, and let $\sigma$ be any map $\sigma$ of $P$ onto a set $R$ with $\|$ as its kernel equivalence: $\sigma p=\sigma q$ iff $p \| q$. The mapping $p \mapsto[p]_{\|}$of $P$ onto the quotient set $P / \|$ is an example. As usual, the preorder $\sqsubseteq$ induces an order relation on $P / \|$ (by $[p]_{\|} \leq[q]_{\|}: \equiv p \sqsubseteq q$ ), which further induces an order on $R$ (by $\sigma(p) \leq \sigma(q): \equiv[p]_{\|} \leq[q]_{\|}$). Clearly, $\sigma$ is then order-preserving. Following (Cīrulis, 2002), we call the obtained poset $R$ a scheme for $P$; the mapping $\varphi$ then can be called a scheme map for $P$.

So, every scheme is a poset isomorphic to $P / \|$, and every scheme map $\sigma$ is characterized by

$$
\sigma(p) \leq \sigma(q) \text { if and only if } p \sqsubseteq q .
$$

Two o-posets $(P, \leq, \sqsubseteq)$ and $\left(P^{\prime}, \leq^{\prime}, \sqsubseteq^{\prime}\right)$ are said to be isomorphic if there is a bijection $h: P \rightarrow P^{\prime}$ such that $p \leq q$ iff $h(p) \leq^{\prime} h(q)$ and $p \sqsubseteq q$ iff $h(p) \sqsubseteq^{\prime} h(q)$ for all $p, q \in P$.

Now consider relations $\sqsubseteq$ and $\|$ on $\Phi$ defined as follows:

$$
\begin{gathered}
(\alpha, \gamma) \sqsubseteq(\beta, \delta): \equiv \alpha \leq \beta \\
(\alpha, \gamma) \|(\beta, \gamma): \equiv \alpha=\beta .
\end{gathered}
$$


Theorem 2.3. The triple $(\Phi, \leq, \sqsubseteq)$ is a tree-ordered o-poset, the mapping $\sigma: \Phi \rightarrow I$ defined by

$$
\sigma(\alpha, \gamma):=\alpha
$$

is a scheme map for $\Phi, \|$ is its kernel equivalence, and I is the corresponding scheme.

Proof. We already know that $\Phi$ is tree-ordered (Lemma 2.2). Clearly $\sqsubseteq$ is a preorder on $\Phi$, and $\|$ is the corresponding equivalence relation. The axiom (i) of overriding is immediately fulfilled. For (ii), suppose that $\left(\alpha_{1}, \gamma_{1}\right) \sqsubseteq\left(\alpha_{2}, \gamma_{2}\right)$ and that $\left(\alpha_{1}, \gamma_{1}\right),\left(\alpha_{2}, \gamma_{2}\right) \leq$ $(\beta, \gamma)$. Then $\gamma_{1}, \gamma_{2} \leq \gamma$, whence $\gamma_{1}$ and $\gamma_{2}$ are comparable ( $O$ is a tree). But $\alpha_{1} \leq \alpha_{2}$ (see (2.3)); as $\left|\alpha_{1}\right|=\left|\gamma_{1}\right|$ and $\left|\alpha_{2}\right|=\left|\gamma_{2}\right|$, it follows that $\left|\gamma_{1}\right| \leq\left|\gamma_{2}\right|$; so $\gamma_{1} \leq \gamma_{2}$ and, further, $\left(\alpha_{1}, \gamma_{1}\right) \leq\left(\alpha_{2}, \gamma_{2}\right)$. For (iii), suppose that $(\alpha, \gamma) \sqsubseteq(\beta, \delta)$. Therefore, $\alpha \leq \beta$ and so $|\alpha| \leq|\beta|=|\delta|$. Clearly, then $(\alpha, \gamma) \|(\alpha, \delta \mid \alpha) \leq(\beta, \delta)$, i.e., the outcome $(\alpha, \delta \mid \alpha)$ is the projection $(\alpha, \gamma) b(\beta, \delta)$. Thus, $\sqsubseteq$ is an overriding relation. The assertions about $\sigma$ and $I$ are evident.

We shall see in Sections 5, 6 that $\Phi$ has even a richer structure.

\section{Virtual states of a black-box automaton}

We adapt the definition of an ND-operator over $[X, Y]$ to black-box automata and call them virtual states of the latter. In this section, let $\mathbf{B}=(I, \varphi, O)$ be a fixed black-box automaton.

Definition 3.1. A virtual state of $\mathbf{B}$ is a function $f: I \rightarrow \mathcal{P}_{0}(\varphi(I))$ such that

- for every $\alpha \in I, f(\alpha) \subseteq \varphi(\alpha)$,

- if $\alpha \leq \beta \in I$, then $f(\alpha)=f(\beta) \mid \alpha$.

A virtual state is deterministic if is a univalent function. We say that a virtual state $f$ is included in a virtual state $g$ (in symbols, $f \subseteq g$ ) if $f(\alpha) \subseteq g(\alpha)$ for all $\alpha \in I$, i.e., if the graph of $f$ is a subset of the graph of $g$.

For instance, the behavior function $\varphi$ of $\mathbf{B}$ is a virtual state. If $\mathbf{B}$ is derived from an ND-automaton $\mathbf{A}$ as in Example of Section 1, then every behavior function $\Lambda_{M}$ is a virtual state of $\mathbf{B}$.

Lemma 3.2 Every virtual state of $\mathbf{B}$ includes a deterministic virtual state.

Proof. Let $f$ be a virtual state. Call a configuration for $f$ any partial function $\omega: I \rightarrow O$ such that

- if $\alpha \in \operatorname{dom} \omega$, then $\omega(\alpha) \in f(\alpha)$,

- if $\alpha \leq \beta \in \operatorname{dom} \omega$, then $\alpha \in \operatorname{dom} \omega$ and $\omega(\alpha)=\omega(\beta) \mid \alpha$.

The function that takes $o$ into $o$ and is undefined elsewhere is an example of such configuration. A total configuration for $f$ is a deterministic virtual state included in $f$, and conversely. It thus remains to demonstrate that total configurations for $f$ exist. Actually, we shall prove more:

every configuration for $f$ is included in a total configuration for $f$. 
Let $\omega_{0}$ be a configuration for $f$. The union of an arbitrary decreasing chain of configurations which include $\omega_{0}$ and are included in $f$ is a configuration of the same kind; so, by Zorn lemma, $\omega_{0}$ is included in a maximal one. But every maximal such configuration is total. Indeed, if a maximal configuration $\omega$ for $f$ is not total, then there is an input element $\alpha \in \operatorname{dom} \omega$ such that $\alpha \leq \beta \in \operatorname{dom} f \backslash \operatorname{dom} \omega$ for some $\beta \in I$. By the finiteness condition in trees, we may assume that $\beta$ is a successor of $\alpha$. Now let $\omega^{\prime}$ be an extension of $\omega$ with $\operatorname{dom} \omega^{\prime}=\operatorname{dom} \omega \cup\{\beta\}$ and such that $\omega^{\prime}(\beta) \in f(\beta)$. This $\omega^{\prime}$ is a configuration for $f$ with $\omega \subset \omega^{\prime}$; so, $\omega$ is not maximal—a contradiction.

Recall that the union of an empty family of sets exists and is, by definition, an empty set. This is why such unions are wxcluded in the following proposition.

Proposition 3.3 The set $V$ of all virtual states is a poset ordered by inclusion and closed under arbitrary non-empty unions. The deterministic states are just its minimal elements, and every virtual state is the union of those deterministic virtual states included in it.

Proof. Of course, the inclusion relation $\subseteq$ is an order relation on $V$. Suppose further that $\left(f_{j}: j \in J\right)$ is a nonempty family of virtual states and that a many-valued mapping $f: I \rightarrow \varphi(I)$ is defined by

$$
\text { for } \alpha \in I, f(\alpha):=\bigcup\left(f_{j}(\alpha): j \in J\right),
$$

As unions preserve the restriction operation $\mid, f$ has all characteristic properties of virtual states.

A deterministic virtual state is always minimal. Conversely, let $f$ be a minimal virtual state of B. By the previous lemma, it includes a deterministic one. Then both states coincide; so $f$ is deterministic.

Therefore, the virtual states form an upper semilattice $(V, \cup, \varphi)$ with $\varphi$ as the greatest element. Let us find out how this semilattice is reflected into the outcome space of B. The next definition is an adaption of Definition 4.1 of (Cīrulis, 2002). We remind that an order ideal (known also as a down or decreasing set) of a poset $P$ is a subset $A$ such that, for all $p, q \in P, q \in A$ whenever $q \leq p \in A$.

Definition 3.4. Let $P$ be an o-poset. An order ideal $A$ of $P$ is said to be

- full if, whenever $p, r \in A, p \sqsubseteq r$ implies that $p \leq q \| r$ for some $q \in A$,

- extensive if to every $p \in P$ there is $q \in A$ such that $p \| q$,

- flat if $p \| q$ for no distinct $p, q \in A$.

The set $P$ is itself an order ideal, which is trivially full and extensive. The following theorem is a variant of Proposition 4.1 in (Cîrulis, 2002), stated there without proof, and is easily proved with help of (2.3) and (2.4).

Theorem 3.5. A subset of $\Phi$ is the graph of a virtual state of $\mathbf{B}$ if and only if it is a full and extensive order ideal. The state is deterministic if and only if the ideal is flat.

Proof. Let $A$ be a subset of $\Phi$, and let $f$ be (possibly, a partial and many-valued) mapping $I \rightarrow \varphi(I)$ having $A$ as its graph. Then 
(i) $\mathrm{A}$ is an order ideal of $\Phi$

iff, for all $(\alpha, \gamma) \in \Phi,(\beta, \delta) \in A$, if $(\alpha, \gamma) \leq(\beta, \delta)$, then $(\alpha, \gamma) \in A$

iff, for all $\alpha, \beta \in I$ and $\gamma \in \varphi(\alpha), \delta \in f(\beta)$, if $\alpha \leq \beta$ and $\gamma \leq \delta$, then $\gamma \in f(\alpha)$

iff, for all $\alpha, \beta \in I$ and $\delta \in f(\beta)$, if $\alpha \leq \beta$, then $\delta \mid \alpha \in f(\alpha)$

iff, for all $\alpha, \beta \in I$, if $\alpha \leq \beta$, then $f(\beta) \mid \alpha \subseteq f(\alpha)$,

(ii) $A$ as an order ideal is full

iff, for all $(\alpha, \gamma),(\beta, \delta) \in A, \alpha \leq \beta$ implies that there is $\left(\beta^{\prime}, \delta^{\prime}\right) \in A$ such that $(\alpha, \gamma) \leq\left(\beta^{\prime}, \delta^{\prime}\right) \|(\beta, \delta)$

iff, for all $\alpha, \beta \in I$ and $\gamma \in f(\alpha), \delta \in f(\beta)$, if $\alpha \leq \beta$, then there are $\beta^{\prime} \in I$ and $\delta^{\prime} \in f\left(\beta^{\prime}\right)$ such that $\alpha \leq \beta^{\prime}, \gamma \leq \delta^{\prime}$ and $\beta^{\prime}=\beta$

iff, for all $\alpha, \beta \in I$ and $\gamma \in f(\alpha)$, if $\alpha \leq \beta$, then, for some $\delta^{\prime} \in f(\beta), \gamma=\delta^{\prime} \mid \alpha$, iff, for all $\alpha, \beta \in I$, if $\alpha \leq \beta$, then $f(\alpha) \subseteq f(\beta) \mid \alpha$,

(iii) $A$ as an order ideal is extensive

iff, to every $(\alpha, \gamma) \in \Phi$, there is $(\beta, \delta) \in A$ such that $(\alpha, \gamma) \|(\beta, \delta)$

iff, to every $\alpha \in I$ and $\gamma \in \varphi(\alpha)$, there are some $\beta \in I$ and $\delta \in f(\beta)$ such that $\alpha=\beta$

iff, to every $\alpha \in I$, there is some $\delta$ in $f(\alpha)$,

(iv) $A$ as an order ideal is flat

iff $(\alpha, \gamma) \|(\beta, \delta)$ for no distinct $(\alpha, \gamma),(\beta, \delta) \in A$

iff, for all $(\alpha, \gamma),(\beta, \delta) \in A$, if $\alpha=\beta$, then $\gamma=\delta$

iff $\gamma=\delta$ for all $\alpha \in I$ and $\gamma \in f(\alpha), \delta \in f(\alpha)$.

It now follows from (i)-(iii) that $A$ is full and extensive order ideal of $\Phi$ if and only if $f$ is a virtual state of $\mathbf{B}$. The other assertion of the theorem follows from (iv).

As $\varphi$ is one of the virtual states of $\mathbf{B}$, it immediately follows that the outcome space $\Phi$ itself is a full and extensive order ideal. Further, the transfer from virtual states to their graphs is bijective and preserves unions. We thus come to the following conclusion.

Corollary 3.6 Full and extensive ideals of the outcome space $\Phi$ form a semilattice isomorphic to the semilattice of virtual states of $\mathbf{B}$.

\section{Back from trees with overriding to black-box automata}

We found out in Section 2 that the outcome space of a black-box automaton ( $b b$ automaton, for short) is a tree-ordered poset with overriding (Theorem 2.3). Let us call any such a poset an $o$-tree. Our aim in this section is to show that, conversely, every o-tree is isomorphic to the outcome o-tree of some black-box automaton.

Lemma 4.1 Let $T$ be a tree, and suppose that

$\tau$ be an order-preserving function from $T$ onto some poset $(R, \leq, 0)$.

If it satisfies the condition

for all $s, t \in T$, if $\tau(s) \leq \tau(t)$, then there is $u \leq t$ such that $\tau(u)=\tau(s)$,

then $R$ is tree-ordered. If $\tau$ is also locally injective, i.e., if

for every $t \in T$ and all $s, s^{\prime} \leq t, s=s^{\prime}$ whenever $\tau(s)=\tau\left(s^{\prime}\right)$,

then $|\tau(t)|=|t|$ for every $t \in T$. 
Proof. The initial supppsition (4.1) implies, in particular, that $\tau(0) \leq \tau(s)$ for any $s \in S$ and, consequently, $\tau(0)=0$.

Assume also (4.2) and consider an initial segment $[0, r]$ of $R$. By surjectivity of $\tau$, $r=\tau(t)$ for some $t \in T$; moreover, for every element $p$ of this segment there is an element $s$ in $T$ such that $p=\tau(s)$. In view of (4.2), we may assume that $s \leq t$. Thus, there is a choice function $m$ which takes every element $p$ of $[0, r]$ into an element of $[0, t] \subseteq T$ so that $\tau(m(p))=p$. Hence, $m$ is injective, and, since the segment $[0, t]$ is finite, the segment $[0, r]$ also has to be finite; more exactly, $|r| \leq|t|$. Further, if $p, p^{\prime} \leq r$, then $m(p), m\left(p^{\prime}\right) \leq t$. But $[0, t]$ is a chain; so, $m(p)$ and $m\left(p^{\prime}\right)$ are comparable. Then $\tau(m(p))$ and $\tau\left(m\left(p^{\prime}\right)\right)$, i.e., $p, p^{\prime}$ are also comparable. Therefore, the segment $[0, r]$ is a chain. Thus $R$ is a tree.

We noticed that $|r| \leq|t|$. If (4.3) also holds, then $\tau$ is injective on $[0, r]$; therefore the reverse inequality also is fulfilled and $|r|=|t|$.

Let us call any function $\tau$ on a tree $T$ with properties (4.1)-(4.3) a projection. For instance, every scheme map for an o-tree is also a projection; see the step (a1) in the proof of the following lemma.

Lemma 4.2 Let $(T, \leq, \sqsubseteq)$ be an tree-ordered poset, and let $\sigma: T \rightarrow L$ be its scheme map. Suppose that given is also a projection $\tau: T \rightarrow R$.

(a) If a function $\varphi: L \rightarrow \mathcal{P}_{0}(R)$ is defined by $\varphi:=\tau \sigma^{-1}$, then $(L, \varphi, R)$ is an exact black-box automaton.

(b) If, in addition, the condition

$$
\text { for all } s, t \in T \text {, if } s \sqsubseteq t \text { and } \tau(s) \leq \tau(t) \text {, then } s \leq t \text {, }
$$

is fulfilled, then $T$ is isomorphic to the outcome o-tree of the automaton.

Proof. (a) We divide the proof of this part of the theorem into several steps.

(a1) Let $T$ be an o-tree with a scheme $L$ and a scheme map $\sigma: T \rightarrow L$. We are going to apply the previous lemma. With $R:=L$ and $\tau:=\sigma,(4.1)$ is evident and the condition (4.2) is fulfilled due to the axiom (iii) of o-posets (Digression 1). Thus $L$ is tree ordered. Next, $\sigma$ is locally injective due to the axiom (ii) of o-posets and (2.2). Thus $|\sigma(t)|=|t|$ by the lemma.

(a2) Further, assume that $\tau$ is one more projection of $T$ onto some poset $(R, \leq, 0)$. Again, Lemma 4.1 applies and $R$ also is a tree; moreover, $|\tau(t)|=|t|$. Together with the equality $|\sigma(t)|=|t|$ obtained in (a1), this yields the equality

$$
|\sigma(t)|=|\tau(t)| \text { for every } t \in S \text {. }
$$

(a3) Next, consider a correspondence $\varphi:=\tau \sigma^{-1}$ between $L$ and $R$; thus, for every $\alpha \in L$,

$$
\varphi(\alpha):=\{\tau(t): \sigma(t)=\alpha\} .
$$

It has the following properties:

(i) $\varphi(\alpha) \neq \varnothing$,

for $\sigma$, being a scheme map onto $L$, is surjective. 
(ii) $\varphi(\alpha) \subseteq R_{\alpha}$ :

if $\gamma \in \varphi(\alpha)$, then $\gamma=\tau(t) \in R$ for some $t$ with $\sigma(t)=\alpha$, but $|\gamma|=|\alpha|$ by (*).

(iii) if $\alpha \leq \beta$ in $L$ and $\gamma \in \varphi(\alpha)$, then $\gamma \leq \delta$ for some $\delta \in \varphi(\beta)$.

First notice that, as $T$ is an o-poset, to every $s^{\prime}$ and $t$ with $s^{\prime} \sqsubseteq t$ there is $t^{\prime} \| t$ such that $s^{\prime} \leq t^{\prime}$. It follows that if $s \sqsubseteq t$ for some $s, t \in T$, then, for every $s^{\prime} \| s$, there is $t^{\prime}$ such that $t^{\prime} \| t$ and $s^{\prime} \leq t^{\prime}$, so, even $\tau\left(s^{\prime}\right) \leq \tau\left(t^{\prime}\right)$.

Now suppose that $\alpha \leq \beta$ and $\gamma \in \varphi(\alpha)$. Choose $s, t \in T$ such that $\alpha=\sigma(s)$ and $\beta=\sigma(t)$; then $s \sqsubseteq t$ (see (2.2)). By the definition of $\varphi$, there is $s^{\prime} \in S$ such that $\gamma=\tau\left(s^{\prime}\right)$ and $\sigma\left(s^{\prime}\right)=\sigma(s)$ (i.e., $\left.s^{\prime} \| s\right)$. As shown in the preceding paragraph, then $t^{\prime} \| t$ and $\tau\left(s^{\prime}\right) \leq \tau\left(t^{\prime}\right)$ for some $t^{\prime}$; hence $\sigma\left(t^{\prime}\right)=\beta$ and $\gamma \leq \tau\left(t^{\prime}\right)$. Put $\delta:=\tau\left(t^{\prime}\right)$; then $\delta \in \varphi(\beta)$ and $\gamma \leq \delta$.

(iv) if $\alpha \leq \beta$ in $L$ and $\delta \in \varphi(\beta)$, then $\gamma \leq \delta$ for some $\gamma \in \varphi(\alpha)$.

Notice again that, as $T$ is an o-poset, if $s \sqsubseteq t^{\prime}$, for some $s, t^{\prime} \in T$, then there is $s^{\prime}$ such that $s \| s^{\prime}$ and $s^{\prime} \leq t^{\prime}$. It follows that if $s \sqsubseteq t$, then, for every $t^{\prime} \| t$ there is $s^{\prime} \| s$ such that $s^{\prime} \leq t^{\prime}$, so, even $\tau\left(s^{\prime}\right) \leq \tau\left(t^{\prime}\right)$.

Now suppose that $\alpha \leq \beta$ and $\delta \in \varphi(\beta)$. Choose $s, t \in T$ such that $\alpha=\sigma(s)$ and $\beta=\sigma(t)$; then $s \sqsubseteq t$ by (2.2). By the definition of $\varphi$, there is $t^{\prime} \in T$ such that $\delta=\tau\left(t^{\prime}\right)$ and $\sigma\left(t^{\prime}\right)=\sigma(t)$ (i.e., $t^{\prime} \| t$ ). As shown in the preceding paragraph, then $s^{\prime} \| s$ and $\tau\left(s^{\prime}\right) \leq \tau\left(t^{\prime}\right)$ for some $s^{\prime}$; hence $\sigma\left(s^{\prime}\right)=\alpha$ and $\varphi\left(s^{\prime}\right) \leq \delta$. Put $\gamma:=\tau\left(s^{\prime}\right)$; then $\gamma \in \varphi(\alpha)$ and $\gamma \leq \delta$.

(v) $\varphi(L)=R$,

because both $\sigma$ and $\tau$ are surjective. In fact, if $\gamma \in \varphi(\alpha)$ for some $\alpha \in L$, then $\gamma \in \tau\left(\sigma^{-1}(\alpha)\right) \subseteq R$. Conversely, if $\gamma \in R$, then $\gamma=\tau(t)$ for some $t \in R$, but $t \in \sigma^{-1}(\alpha)$ for some $\alpha$; so $\gamma \in \varphi(\alpha) \subseteq \varphi(L)$.

We conclude from (i) that $\varphi$ is a function $L \rightarrow \mathcal{P}_{0}(R)$, from (ii), that it satisfies the condition (i) of Definition 2.1, and from (iii) and (iv), that it satisfies also the condition (ii) of the definition. So, $(L, \varphi, R)$ is a black-box automaton, which is exact by (v). Thus (a) is proved.

(b) First notice the following consequence of (4.4):

$$
\text { for all } s, t \in T \text {, if } s \| t \text { and } \tau(s)=\tau(t) \text {, then } s=t .
$$

The outcome space of the constructed automaton is

$$
\begin{aligned}
\Phi & :=\{(\alpha, \gamma): \alpha \in L, \gamma \in \varphi(\alpha)\} \\
& =\{(\sigma(s), \tau(t)): s, t \in T, \tau(t) \in \varphi(\sigma(s))\} \\
& =\{(\sigma(s), \tau(t)): s, t \in T, \sigma(t)=\sigma(s)\} \\
& =\{(\sigma(t), \tau(t)): t \in T\} .
\end{aligned}
$$

As an o-poset, it is isomorphic to the o-poset $T$ : the function $h: t \mapsto(\sigma(t), \tau(t))$ is an isomorphism $T \rightarrow \Phi$. Indeed, it is surjective (by definition), injective (see $(* *))$, and recall that $\sigma$ is a scheme map), $h(\perp)=(o, o)$,

$$
\begin{gathered}
h(s) \leq h\left(s^{\prime}\right) \text { iff }(\sigma(s), \tau(s)) \leq\left(\sigma\left(s^{\prime}\right), \tau\left(s^{\prime}\right)\right) \text { iff } \sigma(s) \leq \sigma\left(s^{\prime}\right) \text { and } \tau(s) \leq \tau\left(s^{\prime}\right) \\
\text { iff } s \sqsubseteq s^{\prime} \text { and } \tau(s) \leq \tau\left(s^{\prime}\right) \text { iff } s \leq s^{\prime}
\end{gathered}
$$


by (2.1), (2.2), (4.4) and the axiom (i) of overriding, while

$$
h(s) \sqsubseteq h\left(s^{\prime}\right) \text { iff }(\sigma(s), \tau(s)) \sqsubseteq\left(\sigma\left(s^{\prime}\right), \tau\left(s^{\prime}\right)\right) \text { iff } \sigma(s) \leq \sigma\left(s^{\prime}\right) \text { iff } s \sqsubseteq s^{\prime}
$$

by (2.3) and (2.2). This completes the proof.

The identity mapping of $T$ onto itself is an instance of a projection $\tau$ in the lemma, which is appropriate for any $T$. Thus the o-trees admit the following representation theorem; according to the proved lemma, the corresponding bb-automaton is $\left(L, \sigma^{-1}, T\right)$.

Theorem 4.3. Every tree-ordered o-poset is isomorphic to the outcome space of an exact black-box automaton.

The next theorem shows that in the case when $T$ itself is an outcome o-poset of a black-box automation, there is another natural choice for $\tau$ and $R$.

Clearly, if a black-box automaton is exact, then it can completely be restored from the "geometrical" structure of its output space: the latter is a set of ordered pairs, and the input and output trees of the automaton are respectively the first and the second projection (in the usual set-theoretic sense) of this set; the behavior function is also determined by the latter. It is seen from the proof of the subsequent theorem that this procedure can actually be viewed as a simple particular case of the general construction of a black-box automaton described in Lemma 4.2. (Recall that a scheme map of a tree-ordered o-poset is its projection map).

Theorem 4.4. Let $(L, \psi, R)$ be an exact black-box automaton and $\Psi$, its outcome otree. The function $\tau: \Psi \rightarrow R$ defined by $(\alpha, \gamma) \mapsto \gamma$ is a projection, and the black-box automaton determined by $\Psi$ and $\tau$ coincides with $(L, \psi, R)$.

Proof. So, assume that $(\Psi, \leq, \sqsubseteq)$ is the outcome o-poset of an exact bb-automaton $(L, \psi, R)$. We already know from Theorem 2.3 that the function $\sigma:(\alpha, \beta) \mapsto \alpha$ is a scheme homomorphism of $T$ onto $L$. Now consider the function $\tau$. Clearly, it is orderpreserving and onto, while the condition (4.2) specializes as follows:

$$
\text { for }(\alpha, \gamma),(\beta, \delta) \in \Psi \text {, if } \gamma \leq \delta \text {, then there is }\left(\alpha^{\prime}, \gamma^{\prime}\right) \leq(\beta, \delta) \text { such that } \gamma^{\prime}=\gamma \text {. }
$$

Suppose that indeed $(\alpha, \gamma),(\beta, \delta) \in \Psi$, and $\gamma \leq \delta$. It follows that $\delta \in \psi(\beta)$ and $|\gamma| \leq$ $|\delta|=|\beta|$. Hence, $\beta \mid \gamma$ exists and $\gamma=\delta \mid \gamma \in \psi(\bar{\beta} \mid \gamma)$. So, the conclusion is fulfilled with $\alpha^{\prime}:=\beta \mid \gamma$ and $\gamma^{\prime}:=\gamma$, and (4.2)holds.

The condition (4.3) takes the form

$$
\text { for all }(\alpha, \gamma),\left(\alpha^{\prime}, \gamma^{\prime}\right) \leq(\beta, \delta) \text {, if } \gamma=\gamma^{\prime} \text {, then }(\alpha, \gamma)=\left(\alpha^{\prime}, \gamma^{\prime}\right) \text {, }
$$

If $(\alpha, \gamma),\left(\alpha^{\prime}, \gamma^{\prime}\right) \leq(\beta, \delta)$, and $\gamma=\gamma^{\prime}$, then $|\alpha|=|\gamma|=\left|\gamma^{\prime}\right|=\left|\alpha^{\prime}\right|$ and, as $\Psi$ is a tree, $(\alpha, \gamma)$ and $\left(\alpha^{\prime}, \gamma\right)$ are comparable; hence, also $\alpha$ and $\alpha^{\prime}$ are comparable in $L$. So $\alpha=\alpha^{\prime}$, and (4.3) is fulfilled.

The condition (4.4) takes the form

$$
\text { for }(\alpha, \gamma),(\beta, \delta) \in \Phi \text {, if } \alpha \leq \beta, \gamma \leq \delta \text {, then }(\alpha, \gamma) \leq(\beta, \delta) \text {. }
$$


and is therefore fulfilled in view of the definition (2.1) of $\leq$ in $\Psi$.

Following the final part of the proof of Theorem 4.2, we now consider a correspondence $\varphi$ between $L$ and $R$ defined for all $\alpha \in L$ by $\varphi(\alpha):=\{\tau(\beta, \delta): \sigma(\beta, \delta)=\alpha\}$. Then immediately, $\varphi(\alpha)=\{\delta:(\beta, \delta) \in \Psi$ and $\beta=\alpha\}=\psi(\alpha)$ (the initial bbautomaton is exact). Thus the constructed black-box automaton $(L, \varphi, R)$ coincides with the original one.

According to this theorem, the transfer from a black-box automaton to its outcome o-tree is reversible: this outcome space completely determines the automaton. On the other hand, the construction of a black-box automaton from a pair $T, \tau$ which was described in Lemma 4.2(a) is also reversible in the weaker sense that the outcome space of the constructed automaton turns out, on an additional condition (4.4), to be isomorphic to the original o-tree $T$. This means that, up to isomorphism, this outcome space does not depend on the choice of the projection $\tau$ (and the scheme map $\sigma$ ). However, we cannot conclude that the constructed automaton itself likewise does not depend, also up to isomorphism, on this choice. It is seen from Theorem 4.4: an analysis of its proof shows that, say, the identity map of $T$ instead of the projection $\tau$ used there (cf. the paragraph preceding Theorem 4.3) leads us to a black-box automaton with the output tree $T$, generally not similar to the original one, i.e., $R$.

Therefore, in spite to the positive results obtained above (in particular, Theorem 4.3), the tree-ordered o-posets turn out to be insufficient for characterizing black-box automata via their outcome spaces. In the next section we deal with richer relational structures which involve two interrelated overriding relations, and show that they do the job.

\section{Repeated look on outcome spaces}

In Lemma 4.2, a scheme of the abstract o-tree $T$ was taken for the income tree of a potential automaton, but, to isolate the output tree of the latter, we had to introduce an additional algebraic device, the projection $\tau$, which generally is outer with respect to the o-tree itself. It will be demonstrated in this section that the projection can be replaced with a certain binary relation living on $T$.

We saw in the preceding section that an scheme map of a tree-ordered o-poset is a projection. The following proposition characterizes connections between scheme and projection maps more fully. Its proof is immediate.

Proposition 5.1 Let $(T, \leq, 0)$ be a tree-ordered poset, $\sqsubseteq$, a binary relation on $T$, and $\tau$, a function $T \rightarrow R$, where $R$ is a poset. The following assertions are equivalent:

(a) $\sqsubseteq$ is an overriding on $T$ and $\tau$ is the corresponding scheme map,

(b) $\tau$ is a projection and $s \sqsubseteq t$ iff $\tau(s) \leq \tau(t)$.

Thus, the projection $\tau$ in Lemma 4.2 implicitly defines one more overriding relation on the o-poset $T$, and is a scheme map for the corresponding o-poset, dual in a sense to the scheme map $\sigma$ for $T$. We thus shall equip a tree, in particular, the outcome set of a black-box automaton, with a pair of interrelated overriding relations. This allows us to obtain a complete characterization of the structure of outcome spaces and smoother representation results for the corresponding abstract algebraic structures. 
Definition 5.2. A double o-poset is a quadruple $\left(P, \leq, \sqsubseteq_{l}, \sqsubseteq_{r}\right)$, where $\left(P, \leq, \sqsubseteq_{l}\right)$ and $\left(P, \leq, \sqsubseteq_{r}\right)$ are o-posets, and

$$
\text { if } p \sqsubseteq_{l} q \text { and } p \sqsubseteq_{r} q \text {, then } p \leq q .
$$

Correspondingly, we now speak about left and right overriding relations $\bigsqcup_{l}$, $\bigsqcup_{r}$, left and right equivalences $\left\|_{l},\right\|_{r}$, left and right scheme maps $\sigma_{l}, \sigma_{r}$ and of respective (left and right) schemes $L, R$ for $P$.

For short, we call a tree-ordered double o-poset a double o-tree.

Every o-poset $(P, \leq, \sqsubseteq)$ can be considered as a double o-poset $(P, \leq, \sqsubseteq,=)$ or either as $(P, \leq,=, \sqsubseteq)$. Theorem 2.3 and Theorem 4.4, together with Proposition 5.1, suggest another example.

Proposition 5.3 Let $(\Phi, \leq)$ be the outcome poset of a black-box automaton $(I, \varphi, O)$. Consider the relations $\Xi_{l}$ and $\Xi_{r}$ on $\Phi$ defined by

$$
(\alpha, \gamma) \sqsubseteq_{l}(\beta, \delta): \equiv \alpha \leq \beta, \quad(\alpha, \gamma) \sqsubseteq_{r}(\beta, \delta): \equiv \gamma \leq \delta .
$$

The system $\left(\Phi, \leq, \sqsubseteq_{l}, \sqsubseteq_{r}\right)$ is a tree-ordered double o-poset, the mappings $\sigma_{l}: \Phi \rightarrow I$, $\sigma_{r}: \Phi \rightarrow O$ defined by

$$
\sigma_{l}(\alpha, \gamma):=\alpha, \quad \sigma_{r}(\alpha, \gamma):=\gamma
$$

being scheme maps for $\Phi$, and $I, O$ are the corresponding schemes.

We say that a double o-poset $\left(P, \leq, \sqsubseteq_{l}, \sqsubseteq_{r}\right)$ is isomorphic to $\left(P^{\prime}, \leq^{\prime}, \sqsubseteq_{l}^{\prime}, \sqsubseteq_{r}^{\prime}\right)$ if there is a function $h: P \rightarrow P^{\prime}$ such that

$$
p \sqsubseteq_{l} q \text { iff } h(p) \sqsubseteq_{l}^{\prime} h(q), \quad p \sqsubseteq_{l} q \text { iff } h(p) \sqsubseteq_{r}^{\prime} h(q)
$$

(then also $p \leq q$ iff $h(p) \leq h(q)$ ) and that a black-box automaton $(I, \varphi, O)$ is isomorphic to an automaton $\left(I^{\prime}, \varphi^{\prime}, O^{\prime}\right)$ if there are order isomorphisms $h_{l}: I \rightarrow I^{\prime}$ and $h_{r}: O \rightarrow O^{\prime}$ such that

$$
\gamma \in \varphi(\alpha) \text { iff } h_{r}(\gamma) \in \varphi^{\prime}\left(h_{l}(\alpha)\right) .
$$

Lemma 5.4 The condition (5.3) is equivalent to any of equalities

$$
h_{r} \varphi=\varphi^{\prime} h_{l}, \varphi^{\prime}=h_{r} \varphi h_{l}^{-1}, \varphi=h_{r}^{-1} \varphi^{\prime} h_{l} .
$$

Proof. First assume that $h_{r} \varphi=\varphi^{\prime} h_{l}$, which means that $\left\{h_{r}(\gamma): \gamma \in \varphi(\alpha)\right\}=$ $\varphi^{\prime}\left(h_{l}(\alpha)\right)$. If $\gamma \in \varphi(\alpha)$, then $h_{r}(\gamma) \in h_{r}(\varphi(\alpha))=\varphi^{\prime}\left(h_{l}(\alpha)\right)$. If, conversely, $h_{r}(\gamma) \in$ $\varphi^{\prime}\left(h_{l}(\alpha)\right)=h_{r}(\varphi(\alpha))$, then there is $\gamma_{1} \in \varphi(\alpha)$ such that $h_{r}(\gamma)=h_{r}\left(\gamma_{1}\right)$. As $h_{r}$ is injective, it follows that $\gamma=\gamma_{1}$, whence $\gamma \in \varphi(\alpha)$. So, (5.3) holds.

Now assume that (5.3) is fulfilled. If $\gamma^{\prime} \in h_{r}(\varphi(\alpha))$, then there is $\gamma \in \varphi(a)$ such that $\gamma^{\prime}=h_{r}(\gamma)$. Hence, $\gamma^{\prime} \in \varphi^{\prime}\left(h_{l}(\alpha)\right)$. Conversely, if $\gamma^{\prime} \in \varphi^{\prime}\left(h_{l}(\alpha)\right)$, then there is $\gamma$ such that $\gamma^{\prime}=h_{r}(\gamma)$, and then $h_{r}(\gamma) \in \varphi^{\prime}\left(h_{l}(\alpha)\right)$. So, $\gamma \in \varphi(\alpha), h_{r}(\gamma) \in h_{r}(\varphi(\alpha))$ and $\gamma^{\prime} \in h_{r}(\varphi(\alpha))$. Thus, $h_{r} \varphi=\varphi^{\prime} h_{l}$.

Therefore, (5.3) is equivalent to the first equality in (5.4). Multiplying both sides of the equality from the left by $h_{l}^{-1}$, we come to the second one; similarly, the second equality yields the first one. Likewise the third equality is equivalent to the first one. 
The 'if' part of the following theorem witnesses that the description of outcome spaces of bb-automata as double o-trees can be considered as full.

Theorem 5.5. Two black-box automata are isomorphic if and only if they outcome spaces are isomorphic as double o-trees.

Proof. Suppose that the mappings $h_{l}: I \rightarrow I^{\prime}, h_{r}: O \rightarrow O^{\prime}$ establish isomorphism of bb-automatons $(I, \varphi, O)$ and $\left(I^{\prime}, \varphi^{\prime}, O^{\prime}\right)$. Consider the function $h: \Phi \rightarrow \Phi^{\prime}$ defined by $h(\alpha, \gamma):=\left(h_{l}(\alpha), h_{r}(\gamma)\right)$. Evidently, it is bijective, as both $h_{l}$ and $h_{r}$ are. Further, $h\left(\alpha_{1}, \gamma_{1}\right) \sqsubseteq_{l} h\left(\alpha_{2}, \gamma_{2}\right)$ iff $h_{l}\left(\alpha_{1}\right) \leq h_{l}\left(\alpha_{2}\right)$ iff $\alpha_{1} \leq \alpha_{2}$ iff $\left(\alpha_{1}, \gamma_{1}\right) \sqsubseteq_{l}\left(\alpha_{2}, \gamma_{2}\right)$, and likewise $h\left(\alpha_{1}, \gamma_{1}\right) \sqsubseteq_{r} h\left(\alpha_{2}, \gamma_{2}\right)$ iff $\left(\alpha_{1}, \gamma_{1}\right) \sqsubseteq_{2}\left(\alpha_{2}, \gamma_{2}\right)$. It follows that $h$ establishes isomorphism of the respective outcome double o-trees $\left(\Phi, \sqsubseteq_{l}, \sqsubseteq_{r}\right)$ and $\left(\Phi^{\prime}, \sqsubseteq_{l}^{\prime}, \sqsubseteq_{r}^{\prime}\right)$.

Now suppose that a function $h: \Phi \rightarrow \Phi^{\prime}$ is a double o-poset isomorphism between the outcome double o-trees of bb-automata $(I, \varphi, O)$ and $\left(I^{\prime}, \varphi^{\prime}, O^{\prime}\right)$. i.e., is bijective and satisfies equivalences

$$
h\left(\alpha_{1}, \gamma_{1}\right) \sqsubseteq_{l}^{\prime} h\left(\alpha_{2}, \gamma_{2}\right) \text { iff } \alpha_{1} \leq \alpha_{2}, \quad h\left(\alpha_{1}, \gamma_{1}\right) \sqsubseteq_{r}^{\prime} h\left(\alpha_{2}, \gamma_{2}\right) \text { iff } \gamma_{1} \leq \gamma_{2}
$$

(see (5.1)). The first one implies that $h\left(\alpha, \gamma_{1}\right) \|_{l}^{\prime} h\left(\alpha, \gamma_{2}\right)$ for all $\alpha \in I$ and $\gamma_{1}, \gamma_{2} \in O$. So, the definition

$$
h_{l}(\alpha)=\alpha^{\prime}: \equiv h(\alpha, \gamma)=\left(\alpha^{\prime}, \gamma^{\prime}\right) \text { for some } \gamma \in I, \gamma^{\prime} \in I^{\prime}
$$

is correct and therefore presents a function $h_{l}: I \rightarrow I^{\prime}$. Indeed, if it happens that $h(\alpha, \gamma)=\left(\alpha^{\prime}, \gamma^{\prime}\right)$ and $h\left(\alpha, \gamma_{1}\right)=\left(\alpha_{1}^{\prime}, \gamma_{1}^{\prime}\right)$, then $h(\alpha, \gamma) \|_{l}^{\prime} h\left(\alpha, \gamma_{1}\right)$, i.e., $\left(\alpha^{\prime}, \gamma^{\prime}\right) \|_{l}^{\prime}$ $\left(\alpha_{1}^{\prime}, \gamma_{1}^{\prime}\right)$, whence $\alpha^{\prime}=\alpha_{1}^{\prime}$.

This function is

- injective: if $h_{l}\left(\alpha_{1}\right)=h_{l}\left(\alpha_{2}\right)$, then there is $\alpha^{\prime} \in I^{\prime}$ such that $h_{l}\left(\alpha_{1}\right)=\alpha^{\prime}=$ $h_{l}\left(\alpha_{2}\right)$, i.e., $h\left(\alpha_{1}, \gamma_{1}\right)=\left(\alpha^{\prime}, \gamma_{1}^{\prime}\right.$ and $h\left(\alpha_{2}, \gamma_{2}\right)=\left(\alpha^{\prime}, \gamma_{2}^{\prime}\right)$ for some $\gamma, \gamma_{1} \in I, \gamma^{\prime}, \gamma_{1}^{\prime} \in$ $I^{\prime}$. As $\left(\alpha^{\prime}, \gamma_{2}^{\prime}\right) \|_{l}^{\prime}\left(\alpha^{\prime}, \gamma_{2}^{\prime}\right)$, then $h\left(\alpha_{1}, \gamma_{1}\right) \|_{l}^{\prime} h\left(\alpha_{2}, \gamma_{2}\right)$, whence $\alpha_{1}=\alpha_{2}$ by $\left.{ }^{*}\right)$;

- surjective: for every $\left(\alpha^{\prime}, \gamma^{\prime}\right) \in I^{\prime}$, there is a pair $(\alpha, \gamma)$ in $\Phi$ such that $h(\alpha, \gamma)=$ $\left(\alpha^{\prime}, \gamma^{\prime}\right)$, whence $h_{l}(\alpha)=\alpha^{\prime}$.

Moreover, $h_{l}$ is an order isomorphism: assuming that $h\left(\alpha_{1}, \gamma_{1}\right)=\left(\alpha_{1}^{\prime}, \gamma_{1}^{\prime}\right)$ and that $h\left(\alpha_{2}, \gamma_{2}\right)=\left(\alpha_{2}^{\prime}, \gamma_{2}^{\prime}\right)$,

$$
\alpha_{1} \leq \alpha_{2} \text { iff }\left(\alpha_{1}^{\prime}, \gamma_{1}^{\prime}\right) \sqsubseteq_{l}\left(\alpha_{2}^{\prime}, \gamma_{2}^{\prime}\right) \text { iff } \alpha_{1}^{\prime} \leq^{\prime} \alpha_{2}^{\prime} \text { iff } h_{l}\left(\alpha_{1}\right) \leq h_{l}\left(\alpha_{2}\right) .
$$

by $(*),(5.1)$ and the definition of $h_{l}$. Likewise, the definition $h_{r}(\gamma)=\gamma^{\prime}: \equiv h\left(\alpha_{1}, \gamma\right)=$ $\left(\alpha_{2}, \gamma^{\prime}\right)$ is correct and presents an order isomorphism $h_{l}: O \rightarrow O^{\prime}$. Finally, notice that $h(\alpha, \gamma)=\left(h_{l}(\alpha), h_{r}(\gamma)\right)$ for all $\alpha, \beta$. Then (5.3) holds:

$$
\begin{aligned}
\gamma \in \varphi(\alpha) \text { iff }(\alpha, \gamma) \in \Phi \text { iff } h(\alpha, \gamma) & \in \Phi^{\prime} \\
& \text { iff }\left(h_{l}(\alpha), h_{r}(\gamma)\right) \in \Phi^{\prime} \text { iff } h_{r}(\gamma) \in \varphi^{\prime}\left(h_{l}(\alpha)\right)
\end{aligned}
$$

Thus, the pair of functions $h_{l}, h_{r}$ establish an isomorphism of the two bb-automata.

The item (a) in the next theorem is a direct analogue, and consequence, of Lemma 4.2. Its item (b) corresponds to Theorem 4.4. 
Theorem 5.6. Let $T:=\left(T, \leq, \sqsubseteq_{l}, \sqsubseteq_{r}\right)$ be a tree-ordered double o-poset.

(a) Suppose that $\sigma_{l}: T \rightarrow L$ is its left scheme map, and $\sigma_{r}: T \rightarrow R$ is its right scheme map. If a mapping $\varphi: L \rightarrow \mathcal{P}_{0}(R)$ is defined by $\varphi:=\sigma_{r} \sigma_{l}^{-1}$, then the triple $(L, \varphi, R)$ is an exact black-box automaton, and $T$ is isomorphic to its outcome double o-tree.

(b) The black-box automaton determined by any other choice of a pair of scheme maps for $T$ is isomorphic to $(L, \varphi, R)$.

(c) Every black-box automaton isomorphic to $(L, \varphi, R)$ arises as in (a) by an appropriate choice of a pair of scheme maps for $T$.

Proof. (a) Assume that $\sigma_{l}, \sigma_{r}$ and $\varphi$ are as in the supposition. We are going to apply Lemma 4.2 with the scheme maps $\sigma_{l}, \sigma_{r}$ standing there for $\sigma$ and $\tau$, respectively. As we know from Proposition 5.1, $\sigma_{r}$ is a projection. So, $(L, \varphi, R)$ is an exact black-box automaton.

The mapping $h$ used in the proof of Theorem 4.2(b) goes also for a double oposet isomorphism from $T$ to the outcome double o-poset of the constructed blackbox automaton (compare the equality (4.4) with the characteristic condition on double o-posets). More accurately, nov $h(t):=\left(\sigma_{l}(t), \sigma_{r}(t)\right)$.

(b) Now assume that $\sigma_{l}^{\prime}: S \rightarrow L^{\prime}$ and $\sigma_{r}^{\prime}: S \rightarrow R^{\prime}$ is another pair of a left and a right scheme maps for $S$ and that $\left(L^{\prime}, \varphi^{\prime}, R^{\prime}\right)$ is the corresponding black-box automaton. By (a), its outcome double o-tree is isomorphic to $T$. By Theorem 5.5, the automaton is then isomorphic to $(L, \varphi, R)$.

(c) Finally, suppose that some black-box automaton $\left(L^{\prime}, \varphi^{\prime}, R^{\prime}\right)$ is isomorphic to $(L, \varphi, R)$, i.e., there are appropriate order isomorphisms $h_{l}: L \rightarrow L^{\prime}$ and $h_{r}: R \rightarrow R^{\prime}$. Then the mapping $\sigma_{l}^{\prime}: T \rightarrow L^{\prime}$ defined by $\sigma_{l}^{\prime}:=h_{l} \sigma_{l}$ is a left scheme map for $T$ with a scheme $L^{\prime}$ :

- the relation $\|_{l}$ is the kernel equivalence of $\sigma_{l}^{\prime}: s_{1} \|_{l} s_{2}$ iff $\sigma_{l}\left(s_{1}\right)=\sigma_{l}\left(s_{2}\right)$ iff $h_{l}\left(\sigma_{l}\left(s_{1}\right)\right)=h_{l}\left(\sigma_{l}\left(s_{2}\right)\right)$.

$-\sigma_{l}^{\prime}$ is surjective as both its components are mappings onto.

In addition, the order which $\sigma_{l}^{\prime}$ as a scheme map induces on $L^{\prime}$ coincides with that already existing on the output tree $L^{\prime}: \sigma_{l}^{\prime}(s) \leq \sigma_{l}^{\prime}(t)$ in $L^{\prime}$ iff $\sigma_{l}(s) \leq \sigma_{l}(t)$ in $L$ (as $h_{l}$ is an order isomorphism) iff $s \sqsubseteq_{l} t$ in $T$ (as $\sigma_{l}$ is a scheme map).

Dually, the mapping $\sigma_{r}^{\prime}: T \rightarrow R^{\prime}$ defined by $\sigma_{r}^{\prime}:=h_{r} \sigma_{r}$ is a right scheme map for $T$ with a scheme $R^{\prime}$.

It remains to check that $\varphi^{\prime}$ is related to $\sigma_{l}^{\prime}$ and $\sigma_{r}^{\prime}$ in a proper way. We already know that $\gamma \in \varphi(\alpha)$ iff $h_{r}(\gamma) \in \varphi^{\prime}\left(h_{l}(\alpha)\right)$, i.e., that $\varphi^{\prime}=h_{r} \varphi h_{l}^{-1}$ Then $\sigma_{r}^{\prime}\left(\sigma_{l}^{\prime}\right)^{-1}=$ $h_{r} \sigma_{r} \sigma_{l}^{-1} h_{l}^{-1}=h_{r} \varphi h_{l}^{-1}=\varphi^{\prime}$ by (5.4), as required.

We say that a bb-automaton is induced by a double o-tree $T$ if it arises from $T$ as in (a) with help of any pair of scheme maps.

Corollary 5.7 Two double o-trees are isomorphic if and only if there is a black-box automaton induced by both of them or, equivalently, if and only if every black-box automaton induced by one of them is induced also by the other. 
Proof. Assume that a bb-automaton B is induced by double o-trees $T$ and $T^{\prime}$. Then they are isomorphic, as, by Theorem 5.6, each of them is isomorphic to the associated double o-tree of $\mathbf{B}$.

Now assume that $T$ is a double o-tree and that a mapping $h$ establishes an isomorphism of it with A double o-tree $\left(T^{\prime}, \leq^{\prime}, \sqsubseteq_{l}^{\prime}, \sqsubseteq_{r}^{\prime}\right)$. Suppose that $\mathbf{B}^{\prime}:=\left(I^{\prime}, \varphi^{\prime}, O^{\prime}\right)$ is an bb-automaton induced by $T^{\prime}$ with help of scheme maps $\sigma_{l}^{\prime}: T^{\prime} \rightarrow I^{\prime}$ and $\sigma_{r}^{\prime}: T \rightarrow O^{\prime}$; thus, $\varphi^{\prime}=\sigma_{r}^{\prime}\left(\sigma_{l}^{\prime}\right)^{-1}$. Then $\sigma_{l}^{\prime} h$ is a left scheme map for $T$. Indeed, for $s, t \in T$,

$$
\left(\sigma_{l}^{\prime} h\right)(s)=\left(\sigma_{l}^{\prime} h\right)(t) \text { iff } \sigma_{l}^{\prime}(h(s))=\sigma_{l}^{\prime}(h(t)) \text { iff } h(s) \|_{l}^{\prime} h(t) \text { iff } s \|_{l} t
$$

(as $\sigma_{l}^{\prime}$ is a left scheme map and $h$ is a double o-poset homomorphism). Dually, $\sigma_{r}^{\prime} h$ is a right scheme map for $T$. Further, $\left(\sigma_{r}^{\prime} h\right)\left(\sigma_{l}^{\prime} h\right)^{-1}=\sigma_{r}^{\prime} h h^{-1}\left(\sigma_{l}^{\prime}\right)^{-1}=\sigma_{r}^{\prime}\left(\sigma_{l}^{\prime}\right)^{-1}=\varphi^{\prime}$. Therefore, $\mathbf{B}^{\prime}$ is induced also by $T$. In fact, every automaton induced by $T^{\prime}$ is similarly induced by $T$ and conversely.

Corollary 5.8 Let $\mathbf{T}$ be a double o-tree and $\mathbf{B}$, a black-box automaton. Then $T$ is isomorphic to the outcome double o-tree of $\mathbf{B}$ if and only if $\mathbf{B}$ is induced by $T$.

Proof. So, assume that $\left(\Phi, \leq, \sqsubseteq_{l}, \sqsubseteq_{r}\right)$ is the outcome space of an automaton $\mathbf{B}:=$ $(L, \varphi, R)$. Let $(L, \psi, R)$ be the induced bb-automaton; so, $\psi$ is defined by $\psi(\alpha):=$ $\left\{\sigma_{r}(\beta, \delta): \sigma_{l}(\beta, \delta)=\alpha\right\}$. Then immediately, $\psi(\alpha)=\{\delta:(\beta, \delta) \in \Phi$ and $\beta=\alpha\}=$ $\psi(\alpha)$. Thus the constructed black-box automaton $(L, \varphi, R)$ coincides with the original one. Now suppose that, conversely, $\mathbf{B}:=(I, \varphi, O)$ is a bb-automaton induced by $T$. By Theorem 5.6(a), $T$ is isomorphic to the associated double o-tree of $\mathbf{B}$.

\section{Outcome space as a bisemigroup}

The outcome spaces of black-box automata admit even a richer algebraic structure. Let $\Phi$ be an outcome space of some automaton $\mathbf{B}$; we are going to consider the binary operations $\circ_{r}, \circ_{l}$ on $\Phi$ defined respectively by

$$
\begin{gathered}
(\alpha, \gamma) \circ_{l}(\beta, \delta):=(\alpha \wedge \beta, \delta \mid(\alpha \wedge \beta)) \\
(\alpha, \gamma) \circ_{r}(\beta, \delta):=(\alpha \mid(\gamma \wedge \delta), \gamma \wedge \delta)
\end{gathered}
$$

These definitions are correct, for $|\alpha \wedge \beta| \leq|\beta|=|\delta|$ and $|\gamma \wedge \beta| \leq|\gamma|=|\alpha|$. To proceed, we need some information on idempotent semigroups (bands).

Digression 2. The natural order $\leq$ of a band $(S, \cdot)$ is defined by $x \leq y: \equiv x y=$ $x=y x$ (equivalently, $y x y=x$ ). An element $0 \in S$ is the zero element, i.e., such that $0 x=0=x 0$, if and only if it is the least element of $S$. The relation $\sqsubseteq$ on $S$ given by $x \sqsubseteq y: \equiv x y x=x$ is reflexive and transitive; we call it the natural preorder of $S$. A band $S$ is said to be right normal if the identity $x y z=y x z$ holds in it. If this is the case, then $x \leq y$ iff $x y=x$ and $x \sqsubseteq y$ iff $y x=x$. The band is called left normal if the operation - satisfies the identity $x y z=x z y$; its natural order is then characterized by the condition $x \leq y$ iff $y x=x$, and its natural preorder, by the condition $x \sqsubseteq y$ iff $x y=x$. Thus there is a full left-right duality between thel bands of these two types. See (Howie, 1995), Section 4.4 for more information on bands. 
It is checked by elementary calculations that the natural preorder of a right normal band $S$ is an overriding relation relatively to the natural order of $S$ : one can take $p r$ for $q$ in the axiom (iii), i.e., $p b r=p r$ in this case. Dually, the natural preorder of a left normal band also is an overriding relation with $p b r=r p$.

A bisemigroup is an algebra with two associative operations. However, if they both are idempotent, the algebra is usually called a double band.

Definition 6.1. We say that a double band $\left(S,{ }_{l}, \cdot_{r}\right)$ is normal if the band $\left(S,{ }^{\prime} l\right)$ is right normal and the band $\left(S, \cdot_{r}\right)$ is left normal. A normal double band is a restrictive bisemigroup (see (Shain, 1965), p. 170) if its pair of operations is associative in the sense that $(x \cdot l y) \cdot_{r} z=x \cdot l(y \cdot r z)$ for all $x, y, z$.

We thus may speak of the left and the right naturtal orders $\leq_{l}, \leq_{r}$ and natural preorders $\sqsubseteq_{l}, \sqsubseteq_{r}$ of a double band. Now we can return to the above operations $\circ_{l}$ and $\mathrm{o}_{l}$.

Theorem 6.2. Let $(\Phi, \leq)$ be the outcome poset of a black-box automaton. The algebra $\left(\Phi, \circ_{r}, \circ_{l}\right)$ is a restrictive semigroup, where both natural orders agree with $\leq$ and the natural preorders satisfy (5.1).

Proof. We first prove that the algebra $\left(\Phi, \circ_{l}\right)$ is a right normal band. The operation $\circ_{l}$ is idempotent: $(\alpha, \gamma) \circ_{l}(\alpha, \gamma)=(\alpha, \gamma \mid \alpha)=(\alpha, \gamma)$. It is also associative:

$$
\begin{gathered}
\left(\left(\alpha_{1}, \gamma_{1}\right) \circ_{l}\left(\alpha_{2}, \gamma_{2}\right)\right) \circ_{l}\left(\alpha_{3}, \gamma_{3}\right)=\left(\alpha_{1} \wedge \alpha_{2}, \gamma_{2} \mid\left(\alpha_{1} \wedge \alpha_{2}\right)\right) \circ_{l}\left(\alpha_{3}, \gamma_{3}\right) \\
=\left(\alpha_{1} \wedge \alpha_{2} \wedge \alpha_{3}, \gamma_{3} \mid\left(\alpha_{1} \wedge \alpha_{2} \wedge \alpha_{3}\right)\right) \\
=\left(\alpha_{1}, \gamma_{1}\right) \circ_{l}\left(\alpha_{2} \wedge \alpha_{3}, \gamma_{3} \mid\left(\alpha_{2} \wedge \alpha_{3}\right)\right)=\left(\alpha_{1}, \gamma_{1}\right) \circ_{l}\left(\left(\alpha_{2}, \gamma_{2}\right) \circ_{l}\left(\alpha_{3}, \gamma_{3}\right)\right) .
\end{gathered}
$$

Likewise,

$$
\begin{gathered}
\left(\left(\alpha_{1}, \gamma_{1}\right) \circ_{l}\left(\alpha_{2}, \gamma_{2}\right)\right) \circ_{l}\left(\alpha_{3}, \gamma_{3}\right)=\left(\alpha_{1} \wedge \alpha_{2} \wedge \alpha_{3}, \gamma_{3} \mid\left(\alpha_{1} \wedge \alpha_{2} \wedge \alpha_{3}\right)\right) \\
=\left(\left(\alpha_{2}, \gamma_{2}\right) \circ_{l}\left(\alpha_{1}, \gamma_{1}\right)\right) \circ_{l}\left(\alpha_{3}, \gamma_{3}\right)
\end{gathered}
$$

i.e., the band $\left(\Phi, \circ_{l}\right)$ is indeed right normal. Evidently, $(o, o)$ is its zero element. Further,

$$
\begin{gathered}
\left(\alpha_{1}, \gamma_{1}\right) \leq_{l}\left(\alpha_{2}, \gamma_{2}\right) \text { iff }\left(\alpha_{1}, \gamma_{1}\right)=\left(\alpha_{1}, \gamma_{1}\right) \circ_{l}\left(\alpha_{2}, \gamma_{2}\right) \\
\text { iff }\left(\alpha_{1}, \gamma_{1}\right)=\left(\alpha_{1} \wedge \alpha_{2}, \gamma_{2} \mid\left(\alpha_{1} \wedge \alpha_{2}\right)\right) \\
\text { iff }\left(\alpha_{1}=\alpha_{1} \wedge \alpha_{2} \text { and } \gamma_{1}=\gamma_{2}\left|\left(\alpha_{1} \wedge \alpha_{2}\right)=\gamma_{2}\right| \alpha_{1}\right) \text { iff } \alpha_{1} \leq \alpha_{2} \text { and } \gamma_{1} \leq \gamma_{2}
\end{gathered}
$$

and

$$
\left(\alpha_{1}, \gamma_{1}\right) \sqsubseteq_{l}\left(\alpha_{2}, \gamma_{2}\right) \text { iff }\left(\alpha_{1}, \gamma_{1}\right)=\left(\alpha_{2}, \gamma_{2}\right) \circ_{l}\left(\alpha_{1}, \gamma_{1}\right) \text { iff } \alpha_{1} \leq \alpha_{2} ;
$$

the relations $\leq_{r}$ and $\sqsubseteq_{r}$ satisfy dual relationships. Thus $\leq_{l}$ and $\leq_{r}$ agree with $\leq$ as required, and (5.1) also holds.

It remains to check the associativity rule for the pair $\circ_{l}, \circ_{r}$. Actually, we shall show that both sides of it reduce to the same expression. At first,

$$
\begin{aligned}
\left(\left(\alpha_{1}, \gamma_{1}\right) \circ_{l}\left(\alpha_{2}, \gamma_{2}\right)\right) \circ_{r}\left(\alpha_{3}, \gamma_{3}\right)=\left(\alpha_{1} \wedge \alpha_{2}, \gamma_{2} \mid\left(\alpha_{1} \wedge \alpha_{2}\right)\right) \circ_{r}\left(\alpha_{3}, \gamma_{3}\right) & \\
= & \left(\left(\alpha_{1} \wedge \alpha_{2}\right) \mid\left(\left(\gamma_{2} \mid\left(\alpha_{1} \wedge \alpha_{2}\right)\right) \wedge \gamma_{3}\right),\left(\gamma_{2} \mid\left(\alpha_{1} \wedge \alpha_{2}\right)\right) \wedge \gamma_{3}\right)
\end{aligned}
$$


Consider two cases. If $\left|\gamma_{2} \wedge \gamma_{3}\right| \leq\left|\alpha_{1} \wedge \alpha_{2}\right|$, then $\left(\gamma_{2} \mid\left(\alpha_{1} \wedge \alpha_{2}\right)\right) \wedge \gamma_{3}=\gamma_{2} \wedge \gamma_{3}$, for $\left(\gamma_{2} \mid\left(\alpha_{1} \wedge \alpha_{2}\right)\right) \wedge \gamma_{3} \leq \gamma_{2} \wedge \gamma_{3}$ and $\gamma_{2} \wedge \gamma_{3}=\gamma_{2}\left|\left(\gamma_{2} \wedge \gamma_{3}\right) \leq \gamma_{2}\right|\left(\alpha_{1} \wedge \alpha_{2}\right)$. Therefore,

$$
\left(\left(\alpha_{1}, \gamma_{1}\right) \circ_{l}\left(\alpha_{2}, \gamma_{2}\right)\right) \circ_{r}\left(\alpha_{3}, \gamma_{3}\right)=\left(\left(\alpha_{1} \wedge \alpha_{2}\right) \mid\left(\gamma_{2} \wedge \gamma_{3}\right), \gamma_{2} \wedge \gamma_{3}\right)
$$

in this case. If, on the contrary, $\left|\gamma_{2} \wedge \gamma_{3}\right| \geq\left|\alpha_{1} \wedge \alpha_{2}\right|$, then $\left(\gamma_{2} \mid\left(\alpha_{1} \wedge \alpha_{2}\right)\right) \wedge \gamma_{3}=$ $\left(\left(\gamma_{2} \wedge \gamma_{3}\right) \mid\left(\alpha_{1} \wedge \alpha_{2}\right)\right) \wedge \gamma_{3}=\left(\gamma_{2} \wedge \gamma_{3}\right) \mid\left(\alpha_{1} \wedge \alpha_{2}\right)$ and

$$
\left(\left(\alpha_{1}, \gamma_{1}\right) \circ_{l}\left(\alpha_{2}, \gamma_{2}\right)\right) \circ_{r}\left(\alpha_{3}, \gamma_{3}\right)=\left(\alpha_{1} \wedge \alpha_{2},\left(\gamma_{2} \wedge \gamma_{3}\right) \mid\left(\alpha_{1} \wedge \alpha_{2}\right)\right) .
$$

On the other hand,

$$
\begin{aligned}
& \left(\alpha_{1}, \gamma_{1}\right) \circ_{l}\left(\left(\alpha_{2}, \gamma_{2}\right) \circ_{r}\left(\alpha_{3}, \gamma_{3}\right)\right)=\left(\alpha_{1}, \gamma_{1}\right) \circ_{l}\left(\alpha_{2} \mid\left(\gamma_{2} \wedge \gamma_{3}\right), \gamma_{2} \wedge \gamma_{3}\right) \\
& \quad=\left(\alpha_{1} \wedge\left(\alpha_{2} \mid\left(\gamma_{2} \wedge \gamma_{3}\right)\right),\left(\gamma_{2} \wedge \gamma_{3}\right) \mid\left(\alpha_{1} \wedge\left(\alpha_{2} \mid\left(\gamma_{2} \wedge \gamma_{3}\right)\right)\right)\right) .
\end{aligned}
$$

If $\left|\gamma_{2} \wedge \gamma_{3}\right| \leq\left|\alpha_{1} \wedge \alpha_{2}\right|$, then $\alpha_{1} \wedge\left(\alpha_{2} \mid\left(\gamma_{2} \wedge \gamma_{3}\right)\right)=\alpha_{1} \wedge\left(\left(\alpha_{1} \wedge \alpha_{2}\right) \mid\left(\gamma_{2} \wedge \gamma_{3}\right)\right)=$ $\left(\alpha_{1} \wedge \alpha_{2}\right) \mid\left(\gamma_{2} \wedge \gamma_{3}\right)$, whence

$$
\left(\alpha_{1}, \gamma_{1}\right) \circ_{l}\left(\left(\alpha_{2}, \gamma_{2}\right) \circ_{r}\left(\alpha_{3}, \gamma_{3}\right)\right)=\left(\left(\alpha_{1} \wedge \alpha_{2}\right) \mid\left(\gamma_{2} \wedge \gamma_{3}\right), \gamma_{2} \wedge \gamma_{3}\right) .
$$

If $\left|\gamma_{2} \wedge \gamma_{3}\right| \geq\left|\alpha_{1} \wedge \alpha_{2}\right|$, then $\alpha_{1} \wedge\left(\alpha_{2} \mid\left(\gamma_{2} \wedge \gamma_{3}\right)\right)=\alpha_{1} \wedge \alpha_{2}$, for $\alpha_{1} \wedge\left(\alpha_{2} \mid\left(\gamma_{2} \wedge \gamma_{3}\right)\right) \leq$ $\alpha_{1} \wedge \alpha_{2}$ and $\alpha_{1} \wedge \alpha_{2}=\alpha_{2}\left|\left(\alpha_{1} \wedge \alpha_{2}\right) \leq \alpha_{2}\right|\left(\gamma_{2} \wedge \gamma_{3}\right)$. Therefore,

$$
\left(\left(\alpha_{1}, \gamma_{1}\right) \circ_{l}\left(\alpha_{2}, \gamma_{2}\right)\right) \circ_{r}\left(\alpha_{3}, \gamma_{3}\right)=\left(\alpha_{1} \wedge \alpha_{2},\left(\gamma_{2} \wedge \gamma_{3}\right) \mid\left(\alpha_{1} \wedge \alpha_{2}\right)\right) .
$$

Thus, the associativity holds, and $S$ is a restrictive bisemigroup.

The obtained bisemigroup $\left(\Phi, \circ_{l}, \circ_{r}\right)$ may be called the outcome bisemigroup of a given black-box-automaton. According to Lemma 2.2, it is tree ordered. Notice that the double o-poset structure of an outcome space (see Proposition 5.3) has been derived in this section from its bisemigroup structure.

\section{References}

Cīrulis, J. (2002) Knowledge representation in Pawlak's information systems: algebraic aspects, in Either, T., Schewe, K. (eds.) Foundations of information and knowledge systems. FOIKS'02, Lect. Notes Comput. Sci. 2284, pp. 250-267.

Howie, J.M. (1995) Fundamentals of semigroup theory, Clarendon Press, Oxford.

Moore, E.F. (1956) Gedanken-experiments on sequential machines. in Shannon, C.E., McCharty, J. (eds), Automata Studies, Princeton Univ. Press, pp. 129-156.

Starke, P.H. (1969) Abstracte Automaten, WEB, Berlin.

Shain, B.M. (1965) Restrictive bisemigroups (Russian), Izv. Vyss. Uchebn. Zav. Mat. 44. N.1, pp. $168-179$.

Received October 6, 2021 , accepted October 29, 2021 